\title{
Post-operative outcomes associated with Boston type 1 keratoprosthesis implantation in Northeast China
}

\author{
MINGHONG GAO*, YINGXIN CHEN*, JING WANG and CUIYU WANG \\ Department of Ophthalmology, The General Hospital of Shenyang Military Area Command, \\ Shenyang, Liaoning 110840, P.R. China
}

Received April 28, 2018; Accepted October 26, 2018

DOI: $10.3892 /$ etm.2018.6994

\begin{abstract}
The aim of the present study was to investigate the post-operative outcomes associated with Boston type 1 keratoprosthesis (Kpro-1) implantation in the treatment of patients with corneal blindness in Northeast China. Clinical data of patients who had undergone Kpro-1 implantation between July 2010 and November 2014 were retrospectively collected. The visual performance, implant retention and post-surgical complications were recorded for each patient. A total of 20 patients (20 eyes) with corneal blindness were included in the study. Prior to surgery, the patients exhibited poor vision and decreased levels of light perception. At 3 days, 1 month, 6 months, 1 year and 2 years post-surgery, logarithm of the minimum angle of resolution values were significantly decreased compared with the pre-operative values. The initial Kpro-1 implants were retained in 16 eyes. Regarding the post-operative complications, six patients exhibited retroprosthetic membrane formation, two patients presented with endophthalmitis, two patients developed secondary glaucoma, two patients experienced optical cylinder detachment, two patients presented with corneal melting, three patients had retinal detachment, three patients developed corneal ulcers and one patient had secondary optic neuropathy. Overall, the results of the present study suggested that implantation with Kpro-1 may represent an alternative therapeutic strategy for patients following previously failed keratoplasty in Northeast China. Serious complications associated with Kpro-1 implantation are common, and thus, suitable patient selection, continuous follow-up and early treatment interventions are recommended.
\end{abstract}

Correspondence to: Dr Yingxin Chen, Department of Ophthalmology, The General Hospital of Shenyang Military Area Command, 83 Wenhua Road, Shenyang, Liaoning 110840, P.R. China

E-mail: chenyingxin88@yeah.net

*Contributed equally

Key words: corneal blindness, Boston type 1 keratoprosthesis, visual acuity, penetrating keratoplasty

\section{Introduction}

According to the statistics of the World Health Organization in 2012, 4.9 million individuals suffered from bilateral corneal blindness worldwide, which accounted for $\sim 12 \%$ of all cases of blindness (1-3). It has been well established that penetrating keratoplasty (PK) may significantly improve post-surgical visual acuity of patients with corneal blindness; however, the long-term graft survival rates have been estimated to be $28-55 \%$ over 5 years and $11 \%$ at 10 years post-graft implantation in patients treated with repeated keratoplasty (4-7). Furthermore, PK has been reported to be associated with poor post-surgical visual outcomes, as well as surgical complications and/or failure in high-risk patients, including those suffering from aniridia, herpetic keratitis, severe chemical burns and cicatrising diseases (8-11). Therefore, a novel strategy for the treatment of patients with PK and poor prognoses was required, which led to the development of the keratoprosthesis (Kpro) implantation strategy (12).

Boston type 1 Kpro (Kpro-1) is considered to represent an alternative therapeutic strategy for patients suffering from late-stage corneal blindness, which is not specified in standard keratoplasty. Several previous studies have investigated the symptoms, complications and post-surgical outcomes associated with Kpro-1 implantation (13-21); however, the majority of these studies were performed in the USA. Thus far, there has been limited research regarding the evaluation of Kpro implantation outside North America. To the best of our knowledge, only one study investigating the clinical outcomes associated with Kpro-1 implantation in Southern China has been published (22). Therefore, it is important that symptoms, clinical outcomes and complications associated with Kpro-1 implantation in patients in Northeast China are further investigated in future studies.

\section{Materials and methods}

Patients. Clinical data of patients with corneal blindness who had undergone implantation with Kpro-1 between July 2010 and November 2014 were collected in a retrospective manner. Patients were qualified for inclusion if they: i) had a history of PK surgical failure; ii) were not amenable to standard keratoplasty; iii) had no optic neuropathy or retinal neuropathy; and iv) had a good eyelid contour with normal eye blink. Cases with 
autoimmune diseases, including ocular cicatricial pemphigoid or Stevens-Johnson syndrome, were excluded from the present study. Written informed consent was obtained from each patient prior to surgery. The clinical and personal data of the patients were recorded, including age, sex, pre-operative diagnoses, symptoms, prior history of ocular surgeries, ocular and medical comorbidities and visual outcomes.

Surgical technique. A pre-operative assessment of the complete personal medical and family ocular history for each patient was performed. Each patient was subjected to ophthalmological examination to determine whether they were suitable for keratoplasty. The KPro-1 threadless design was purchased from the Massachusetts Eye and Ear Infirmary (Boston, MA, USA). Implantation with Kpro-1 was performed using a standard technique that has been previously described $(23,24)$. Surgical procedures were performed by the same experienced surgeon. A contact lens was placed on the cornea of all patients following KPro implantation. During the post-operative follow-up period, contact lenses were adjusted, replaced and cleaned if required.

Patients' bandages were removed a total of $24 \mathrm{~h}$ post-surgery. Topical tropicamide eye drops (twice per day), levofloxacin eye drops (four times per day), prednisolone eye drops (four times per day) and tobramycin dexamethasone eye ointment (once per night) were administered over a 1-month time period. Following this, the frequency of topical eye drops administered to patients was reduced by half over a 6 -month period. For patients that exhibited elevated intraocular pressure (IOP) post-surgery, loteprednol and anti-glaucoma eye drops, e.g. brimonidine tartrate, were administered twice per day. Following this, patients were subjected to systemic administration of numerous drugs for 6 months, including oral administration of minocycline (twice per day) and a liver-preserving drug as antifungal drugs may result in liver damage.

Outcome measures. Patients were subjected to follow-up examination at the following time intervals post-surgery: 3 days, 1 month, 6 months and every 1 to 3 months thereafter. At each follow-up examination, slit-lamp examination was performed, the logarithm of the minimum angle of resolution (LogMAR), IOP, optic disc and fundus were evaluated, and any possible complications were screened for. The post-operative characteristics were subsequently observed, including best spectacle-corrected visual acuity, device retention and post-operative medications. The incidence of post-operative complications, the timing of such complications and therapeutic regimens administered were also recorded.

Statistical analysis. Quantitative data are expressed as the mean \pm standard deviation and differences between groups were analyzed using one-way analysis of variance followed by Tukey's post-hoc test. Data analysis was performed using SPSS 19.0 software (IBM Corp., Armonk, NY, USA). P<0.05 was considered to indicate a statistically significant difference.

\section{Results}

Baseline characteristics. A total of 20 patients were included in the present study (14 males and 6 females; mean age, $47.15 \pm 14.79$ years; age range, $21-79$ years). Of these patients,
17 cases had a history of failed PK, one had experienced three failed PK surgeries and only two patients had no history of failed PK. All patients were followed up at 3 days, 1 month, 6 months, 1 year and 2 years post-surgery. Details on the patients who received a KPro-1 implant are presented in Table I. Representative mages of the patients' corneas prior to and post-implantation with Kpro-1 are presented in Fig. 1.

Visual acuity. Patients exhibited poor vision and decreased levels of light perception prior to surgery. Following Kpro-1 implantation, visual improvement was observed in all 20 eyes (100\%). The LogMAR values obtained at 3 days, 1 month, 6 month, 1 year and 2 year post-surgery were significantly decreased compared with the pre-operative values $(\mathrm{P}<0.05$; Fig. 2$)$.

Prosthesis retention. The mean follow-up period was $2.89 \pm 1.56$ years post-implantation (range, 3-5 years). During the follow-up period, the initial Kpro-1 was retained in 16 eyes (80\%). In addition, corneal images (Fig. 1) and swept-source optical coherence tomography imaging (Fig. 3) revealed that the artificial and donor corneas in 14 patients were completely healed 1 year following Kpro-1 implantation.

Complications and management. Post-operative complications exhibited by patients are listed in Table II. A total of seven patients $(35 \%)$ experienced one or more post-operative complications. A total of six patients developed retroprosthetic membrane formation (RPM) and mild-to-moderate visual loss. In these six patients, five eyes affected were treated using a neodymium-doped yttrium aluminum garnet (YAG) laser, and one eye was treated via surgical membrane removal; all eyes subsequently exhibited visual improvement. Optical cylinder detachment was observed in two patients at 1- and 2-year time intervals post-surgery, and their LogMAR values were slightly decreased to 0.25 and 1.85 , respectively. Corneal ulcers occurred in three patients; however, inflammation was effectively managed and the visual acuity was improved following administration of active systemic and local anti-infective treatment. Retinal detachment occurred in two patients at 1 and 4 years post-operation. The retina was repositioned in one patient following vitrectomy, retinal reposition and silicone oil filling; the other patient also developed secondary endophthalmitis, however, this was subsequently managed via conservative and surgical treatment. Corneal melting occurred in two patients: One patient refused to undergo a second KPro implantation and the other patient received ophthalmectomy due to additional infective endophthalmitis. In total, endophthalmitis was observed in two patients. Secondary optic neuropathy occurred in one patient at a total of 4 years post-surgery; however, following 3 days of systemic hormone shock therapy combined with oral hormone sequential therapy, the patient's visual acuity remained at 0.2. Secondary glaucoma was detected in two patients. Following the administration of cyclocryotherapy and compound trabeculectomy to these patients, the IOP of the two eyes returned to normal.

\section{Discussion}

PK has provided patients with diseased, opacified or otherwise compromised corneas with a chance to regain sight. Although 
Table I. Details of patients implanted with a KPro-1.

\begin{tabular}{|c|c|c|c|}
\hline Age (years)/sex & Ocular diagnosis & $\begin{array}{c}\text { Number of previous } \\
\text { failed PK }\end{array}$ & Technique \\
\hline $49 / \mathrm{F}$ & Mycotic corneal ulcer & 1 & KPro- $1+$ iridectomy $+\mathrm{AV}$ \\
\hline $36 / \mathrm{M}$ & Leukoma & 1 & KPro-1 + ECCE \\
\hline $23 / \mathrm{M}$ & Mycotic corneal ulcer & 1 & KPro-1 + cataract removal + ILI \\
\hline $21 / \mathrm{M}$ & Alkali burn & 1 & KPro-1 + AV \\
\hline 49/M & Mycotic corneal ulcer & 1 & KPro- $1+$ AV \\
\hline $27 / \mathrm{M}$ & Bacterial corneal ulcer & 1 & KPro-1 \\
\hline $40 / \mathrm{M}$ & Acid burn & 3 & KPro-1 \\
\hline $79 / \mathrm{F}$ & Herpes simplex corneal ulcer & 1 & KPro- $1+$ AV \\
\hline $54 / \mathrm{M}$ & Leukoma + glaucoma & 1 & KPro-1 + CT + cyclocryotherapy \\
\hline $37 / \mathrm{M}$ & Mycotic corneal ulcer & 1 & KPro-1 \\
\hline $42 / \mathrm{M}$ & Mycotic corneal ulcer & 1 & KPro-1 + ECCE + ILI \\
\hline $51 / \mathrm{M}$ & Leukoma & & KPro-1 + ECCE \\
\hline $53 / \mathrm{F}$ & Mycotic corneal ulcer & 1 & KPro-1 + ECCE \\
\hline $45 / \mathrm{M}$ & Mycotic corneal ulcer + Mooren's ulcer & 1 & KPro-1 x2 \\
\hline $56 / \mathrm{F}$ & Mycotic corneal ulcer & 1 & KPro-1 \\
\hline $55 / \mathrm{M}$ & Leukoma & 1 & KPro-1+ AV \\
\hline $61 / \mathrm{F}$ & Glaucoma + CED & & KPro-1 + AV \\
\hline $65 / F$ & Leukoma + glaucoma & 1 & KPro-1 + iridectomy + AV \\
\hline $64 / M$ & Alkali burn & 1 & KPro-1 + AV \\
\hline $36 / \mathrm{M}$ & Viral corneal ulcer & 1 & KPro- 1 + cataract removal + ILI \\
\hline
\end{tabular}

PK, penetrating keratoplasty; CED, corneal endothelial decompensation; CT, compound trabeculectomy; ECCE, extracapsular cataract extraction; ILI, intraocular lens implantation; AV, anterior vitrectomy; M, male; F, female; Kpro-1, Boston type 1 keratoprosthesis.
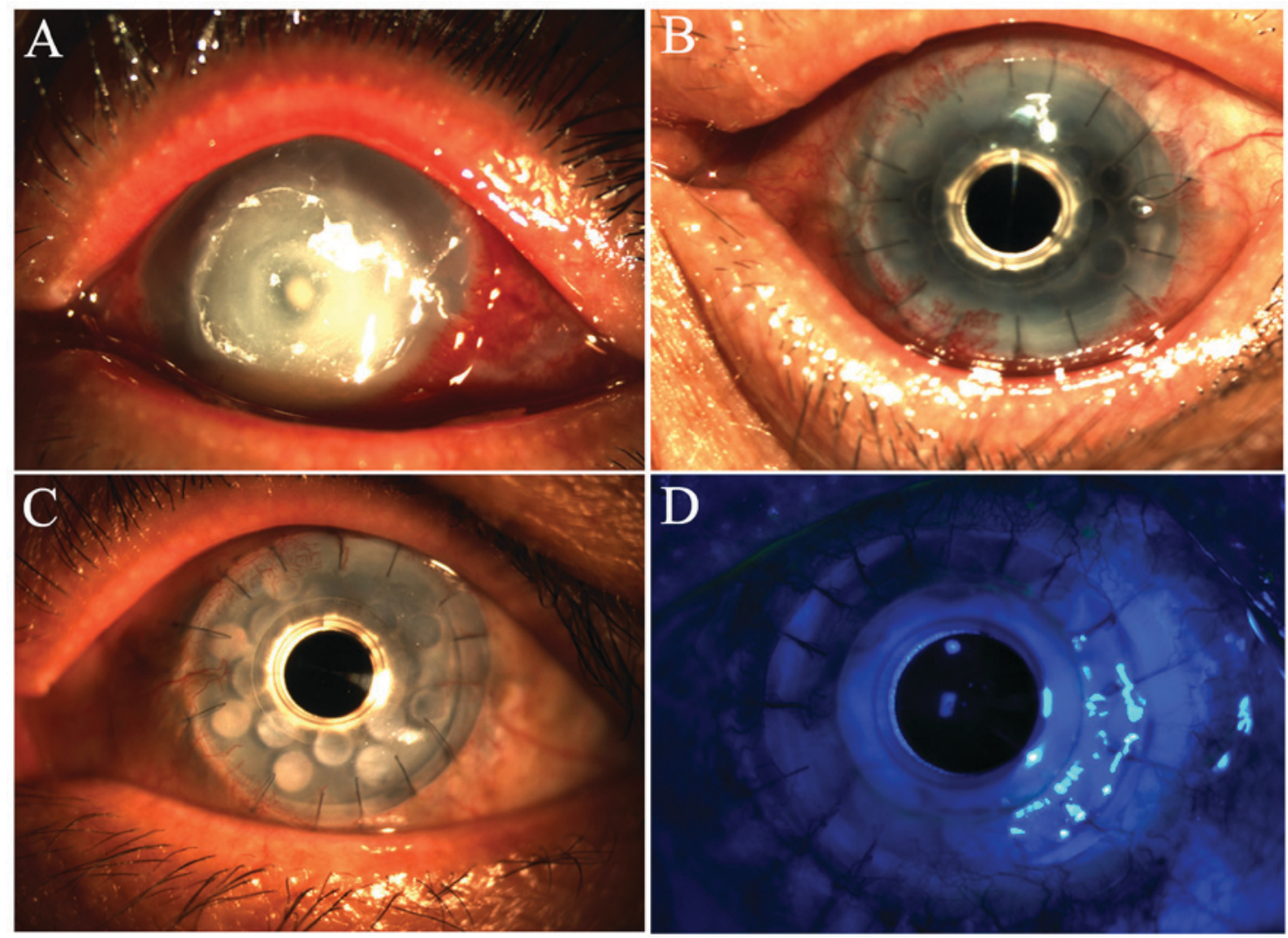

Figure 1. Corneal images from one patient with corneal blindness. (A) Prior to surgery, and at (B) 1 month and (C) 1 year post-implantation with a Boston type 1 keratoprosthesis. (D) Fluorescein staining at 2 years post-surgery revealed that there was no significant staining in the conjunctiva. 


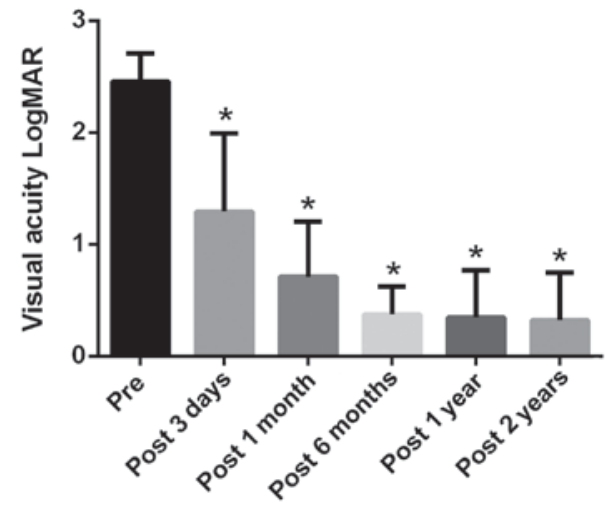

Figure 2. Visual acuity of the patients over a 2-year follow-up period. ${ }^{*} \mathrm{P}<0.05$ vs. Pre. LogMAR, logarithm of the minimum angle of resolution; Pre, pre-surgery; Post, post-surgery.

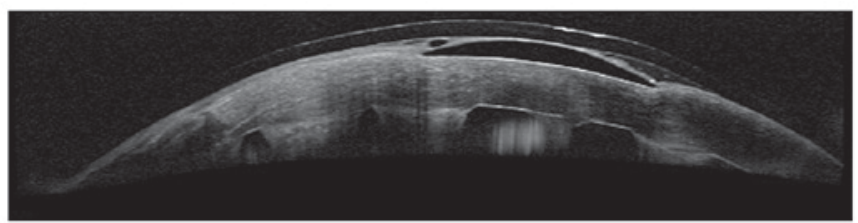

Figure 3. At 1 year post-implantation with Boston type 1 keratoprosthesis, the swept-source optical coherence tomography image of the anterior segment of one patient demonstrated that the corneal epithelium and the complex were fully healed.

this procedure represents the primary treatment option for such conditions, graft failure is a huge obstacle for surgical success. Despite the development of numerous novel drugs and increasingly advanced surgical techniques, $\sim 40 \%$ of patients with PK have been reported to exhibit immunological rejection following corneal grafts over a 10 -year follow-up period, and in half of these cases, this results in graft failure $(7,8,25,26)$. The success rate of secondary corneal graft surgery is markedly lower (4).

Specific indications for Kpro implantation include PK surgical failure, chemical burns and severe corneal scarring vascularization $(27,28)$. To identify patients that may be suitable for Kpro implantation, surgeons should consider causes of visual loss, lid position, ocular surface condition, and concomitant ocular and systemic diseases (29). In addition, Farias et al (30) demonstrated the successful use of intraocular videoendoscopic examination in the pre-operative evaluation of candidates for KPro implantation.

In the present study, the results revealed that the majority of patients exhibited a significant improvement in post-operative visual acuity following KPro implantation due to the removal of retro-KPro membranes and cataracts. Other factors resulting in poor eyesight following artificial cornea transplantation included secondary glaucoma with high intraocular pressure, vitreous opacity and optical cylinder detachment.

The incidence of RPM in the present study was $30 \%$. A previous study by Stacy et al (31) reported an RPM incidence of 25-65\% following Boston type 1 implantation and $45 \%$ of those cases affected required further treatment, including YAG laser treatment or surgical membranectomy. In the present study, two patients presented with secondary glaucoma
Table II. Post-operative complications.

Complication

Cases

Retroprosthetic membrane formation 6

Endophthalmitis

2

Secondary glaucoma

2

Optical cylinder detachment

2

Corneal melting

2

Retinal detachment

2

Corneal ulcer

3

Secondary optic neuropathy

after the implantation. The development and progression of glaucoma in patients with KPro implants may be due to a compromised angle as a result of a crowded anterior chamber, routine topical steroid therapy or debris accumulation in the trabecular meshwork (22). In the present study, endophthalmitis occurred in two patients, which represents one of the most damaging and challenging complications associated with KPro surgery (32). Retinal detachment was observed in two patients of the present study. Patients with underlying autoimmune systemic disorders should be closely monitored for the development of retinal detachment following KPro surgery (33). In the present study, corneal melting occurred in two patients $(10 \%)$, the rate of which was decreased compared with that reported by Cade et al (34). A recent study by Kammerdiener et al (35) suggested that the absence of lens implantation following KPro surgery may increase the risk of post-operative complications, including corneal melting. Therefore, extensive forniceal reconstruction performed to retain the lens may contribute to prolonged retention of the implanted KPros in patients with late-stage corneal blindness.

Of note, the present study had certain limitations. First, the study lacked a concurrent control group and follow-up data of 3 months post-surgery. Furthermore, all procedures in the present study were performed by a single surgeon. Therefore, no data were available for comparison between different surgical procedures or different surgeons. In addition, the size of the study population and the quantity of available OCT data were small. Therefore, future large-scale studies with long-term follow-up periods are required.

In conclusion, the results of the present study suggested that Kpro-1 represents an alternative therapeutic strategy for patients with previously failed keratoplasty in Northeast China. Serious complications are common, and thus, suitable patient selection, continuous follow-ups and early treatment interventions are recommended.

\section{Acknowledgements}

Not applicable.

\section{Funding}

The present study was partly funded by the China Postdoctoral Science Fund (grant no. 2015M572800) and the Natural 
Science Foundation of Liaoning Province in China (grant no. 20170540978).

\section{Availability of data and materials}

The data used to support the results of this study are available from the corresponding author upon request.

\section{Authors' contributions}

MG was a major contributor in the writing of the manuscript and performed the surgery. YC designed the present study and performed literature searches. JW analysed and interpreted the patient data. CW collected and assembled the data. All authors read and approved the final manuscript.

\section{Ethics approval and consent to participate}

This study was approved by the ethical committee of the General Hospital of Shenyang Military Area Command (approval no. 2010L05; Shenyang, China).

\section{Patient consent for publication}

Written informed consent was obtained from all patients prior to surgery, including for the use of personal information for research purposes.

\section{Competing interests}

The authors declare that they have no competing interests.

\section{References}

1. Oliva MS, Schottman T and Gulati M: Turning the tide of corneal blindness. Indian J Ophthalmol 60: 423-427, 2012

2. Pascolini D and Mariotti SP: Global estimates of visual impairment: 2010. Br J Ophthalmol 96: 614-618, 2012.

3. Resnikoff S, Pascolini D, Etya'ale D, Kocur I, Pararajasegaram R, Pokharel GP and Mariotti SP: Global data on visual impairment in the year 2002. Bull World Health Organ 82: 844-851, 2004.

4. Beckingsale P, Mavrikakis I, Al-Yousuf N, Mavrikakis E and Daya SM: Penetrating keratoplasty: Outcomes from a corneal unit compared to national data. Br J Ophthalmol 90: 728-731, 2006.

5. Bersudsky V, Blum-Hareuveni T, Rehany U and Rumelt S: The profile of repeated corneal transplantation. Ophthalmology 108: 461-469, 2001.

6. Ma JJ, Graney JM and Dohlman CH: Repeat penetrating keratoplasty versus the Boston keratoprosthesis in graft failure. Int Ophthalmol Clin 45: 49-59, 2005.

7. Muraine M, Sanchez C, Watt L, Retout A and Brasseur G: Long-term results of penetrating keratoplasty. A 10-year-plus retrospective study. Graefes Arch Clin Exp Ophthalmol 241: 571-576, 2003.

8. Ing JJ, Ing HH, Nelson LR, Hodge DO and Bourne WM: Ten-year postoperative results of penetrating keratoplasty. Ophthalmology 105: 1855-1865, 1998.

9. Kremer I, Rajpal RK, Rapuano CJ, Cohen EJ and Laibson PR: Results of penetrating keratoplasty in aniridia. Am J Ophthalmol 115: 317-320, 1993.

10. Tugal-Tutkun I, Akova YA and Foster CS: Penetrating keratoplasty in cicatrizing conjunctival diseases. Ophthalmology 102: 576-585, 1995.

11. Yaghouti F, Nouri M, Abad JC, Power WJ, Doane MG and Dohlman CH: Keratoprosthesis: Preoperative prognostic categories. Cornea 20: 19-23, 2001

12. Barber JC: Keratoprosthesis: Past and present. Int Ophthalmol Clin 28: 103-109, 1988.
13. Aldave AJ, Sangwan VS, Basu S, Basak SK, Hovakimyan A, Gevorgyan O, Kharashi SA, Jindan MA, Tandon R, Mascarenhas J, et al: International results with the Boston type I keratoprosthesis. Ophthalmology 119: 1530-1538, 2012.

14. Chew HF, Ayres BD, Hammersmith KM, Rapuano CJ, Laibson PR, Myers JS, Jin YP and Cohen EJ: Boston keratoprosthesis outcomes and complications. Cornea 28: 989-996, 2009.

15. Ciolino JB, Belin MW, Todani A, Al-Arfaj K, Rudnisky CJ and Boston Keratoprosthesis Type 1 Study Group: Retention of the Boston keratoprosthesis type 1: Multicenter study results. Ophthalmology 120: 1195-1200, 2013.

16. Dunlap K, Chak G, Aquavella JV, Myrowitz E, Utine CA and Akpek E: Short-term visual outcomes of Boston type 1 keratoprosthesis implantation. Ophthalmology 117: 687-692, 2010.

17. Greiner MA, Li JY and Mannis MJ: Longer-term vision outcomes and complications with the Boston type 1 keratoprosthesis at the University of California, Davis. Ophthalmology 118: 1543-1550, 2011.

18. Kamyar R, Weizer JS, de Paula FH, Stein JD, Moroi SE, John D, Musch DC and Mian SI: Glaucoma associated with Boston type I keratoprosthesis. Cornea 31: 134-139, 2012.

19. Patel AP, Wu EI, Ritterband DC and Seedor JA: Boston type 1 keratoprosthesis: The New York Eye and Ear experience. Eye (Lond) 26: 418-425, 2012.

20. Robert MC and Harissi-Dagher M: Boston type 1 keratoprosthesis: The CHUM experience. Can J Ophthalmol 46: 164-168, 2011.

21. Zerbe BL, Belin MW, Ciolino JB and Boston Type 1 Keratoprosthesis Study Group: Results from the multicenter Boston Type 1 Keratoprosthesis Study. Ophthalmology 113: 1779 e1-7, 2006.

22. Gu J, Zhai J, Zhou S and Chen J: Boston keratoprosthesis outcomes in severe ocular chemical burns in Southern China: A retrospective study. Adv Ther 33: 760-773, 2016.

23. Dohlman $\mathrm{CH}$, et al: Introduction to the use of the Boston keratoprosthesis. Expert Rev Ophthalmology 1: 41-48, 2006.

24. Aquavella JV, Qian Y, McCormick GJ and Palakuru JR: Keratoprosthesis: The Dohlman-Doane device. Am J Ophthalmol 140: 1032-1038, 2005.

25. Sellami D, Abid S, Bouaouaja G, Ben Amor S, Kammoun B, Masmoudi M, Dabbeche K, Boumoud H, Ben Zina Z and Feki J: Epidemiology and risk factors for corneal graft rejection. Transplant Proc 39: 2609-2611, 2007.

26. Price FW and Price MO: Adult keratoplasty: Has the prognosis improved in the last 25 years? Int Ophthalmol 28: 141-146, 2008.

27. De La Paz MF, De Toledo JÁ, Charoenrook V, Sel S, Temprano J, Barraquer RI and Michael R: Impact of clinical factors on the long-term functional and anatomic outcomes of osteo-odonto-keratoprosthesis and tibial bone keratoprosthesis. Am J Ophthalmol 151: 829-839.e1, 2011.

28. Muñoz-Gutierrez G, Alvarez de Toledo J, Barraquer RI, Vera L, Couto Valeria R, Nadal J and de la Paz MF: Post-surgical visual outcome and complications in Boston type 1 keratoprosthesis. Arch Soc Esp Oftalmol 88: 56-63, 2013 (In English, Spanish).

29. Williamson SL and Cortina MS: Boston type 1 keratoprosthesis from patient selection through postoperative management: A review for the keratoprosthetic surgeon. Clin Ophthalmol 10: 437-443, 2016.

30. Farias CC, Ozturk HE, Albini TA, Berrocal AM, Amescua G, Betancurt C, Parel JM, Oliveros MC, Gibbons A, Vargas JM and Perez VL: Use of intraocular video endoscopic examination in the preoperative evaluation of keratoprosthesis surgery to assess visual potential. Am J Ophthalmol 158: 80-86.e2, 2014.

31. Stacy RC, Jakobiec FA, Michaud NA, Dohlman CH and Colby KA: Characterization of retrokeratoprosthetic membranes in the Boston type 1 keratoprosthesis. Arch Ophthalmol 129: 310-316, 2011.

32. Robert MC, Moussally K, and Harissi-Dagher M: Review of endophthalmitis following Boston keratoprosthesis type 1 . Br J Ophthalmol 96: 776-780, 2012.

33. Jardeleza MS, Rheaume MA, Chodosh J, Lane AM and Dohlman CH: Retinal detachments after Boston Keratoprosthesis: Incidence, predisposing factors, and visual outcomes. Digit J Ophthalmol 21: 1-15, 2015.

34. Cade F, Grosskreutz CL, Tauber A and Dohlman CH: Glaucoma in eyes with severe chemical burn, before and after keratoprosthesis. Cornea 30: 1322-1327, 2011.

35. Kammerdiener LL, Speiser JL, Aquavella JV, Harissi-Dagher M, Dohlman CH, Chodosh J and Ciolino JB: Protective effect of soft contact lenses after boston keratoprosthesis. Br J Ophthalmol 100: 549-552, 2016. 